\title{
The LHCf experiment: status and perspectives
}

\author{
${\text { A. } \text { Tricomi }^{* a}, \text { O. Adriani }}^{b}$, L. Bonechi ${ }^{b}$, M. Bongi $^{c}$, G. Castellini $^{d}$, R. D'Alessandro $^{b}$, D. \\ A. Faus ${ }^{e}$, K. Fukui ${ }^{f}$, M. Hagenauer ${ }^{g}$, Y. Itow ${ }^{f}$, K. Kasahara ${ }^{h}$, D. Macina ${ }^{i}$, T. Mase ${ }^{f}$, K. \\ Masuda $^{f}$, Y. Matsubara ${ }^{f}$, H. Menjo ${ }^{f}$, M. Mizuishi ${ }^{h}$, Y. Muraki ${ }^{j}$, P. Papini ${ }^{c}$, A.L. Perrot $^{i}$,

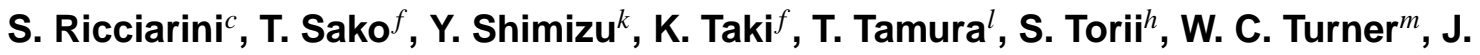 \\ Velasco $^{e}$, A. Viciani ${ }^{c}$, K. Yoshida ${ }^{n}$. \\ ${ }^{a}$ University of Catania and INFN Catania, Italy \\ ${ }^{b}$ University of Firenze and INFN Firenze, Italy \\ ${ }^{c}$ INFN Firenze, Italy \\ ${ }^{d}$ IFAC-CNR and INFN Firenze, Italy \\ ${ }^{e}$ IFIC, Centro Mixto CSIC-UVEG, Valencia, Spain \\ ${ }^{f}$ Solar-Terrestrial Environment Laboratory, Nagoya University, Nagoya, Japan \\ ${ }^{g}$ École-Polytechnique, Paris, France \\ ${ }^{h}$ Research Institute for Science and Engineering, Waseda University, Tokyo, Japan \\ ${ }^{i}$ CERN, Geneva, Switzerland \\ ${ }^{j}$ Konan University, Kobe, Japan \\ ${ }^{k}$ Institute for Cosmic Ray Research, University of Tokyo, Kashiwa, Chiba, Japan \\ ${ }^{l}$ Kanagawa University, Yokohama, Japan \\ ${ }^{m}$ LBNL, Berkeley, California, USA \\ ${ }^{n}$ Shibaura Institute of Technology, Saitama, Japan \\ E-mail: alessia.tricomi@ct.infn.it, lhcf_all@stelab.nagoya-u.ac.jp
}

The LHCf experiment is the smallest of the six LHC experiments and it has been proposed and built as a fully dedicated astroparticle experiment at LHC. The aim of the experiment is indeed to provide a detailed calibration of the Monte Carlo models used in High Energy Cosmic Ray Physics through the measurement of the neutral particles emitted in the very forward region at LHC.

LHCf consists of two calorimeters placed in the LHC tunnel near the ATLAS interaction point. The two calorimeters have also position measurement capabilities thus ensuring the possibility to measure neutral particles in the pseudo-rapidity region greater than eight with execellent performances.

In this paper, the status of the two detectors, their performances and the Physics reach is described.

2008 Physics at LHC

September 29 - 4 October 2008

Split, Croatia

${ }^{*}$ Speaker. 


\section{Introduction}

Understanding the origin of High Energy Cosmic Rays and the high energy phenomena happening in our Universe is strictly related to our capabilities to measure the energy spectrum, the arrival direction and the chemical composition of the cosmic rays reaching our atmosphere. Dedicated extensive air shower experiments are in place since many years and have strongly contributed to our understanding of High and Ultra High Energy Cosmic (UHECR) Ray Physics. However, the results of these experiments are in some cases not fully in agreement and, in addition, the interpretation of their data in terms of primary cosmic ray properties is strongly affected by the knowledge of the nuclear interactions in the earth's atmosphere. This is true, for instance, for the interpretation of the behaviour of the energy spectrum in the UHE region, in particular the existence of events above the so called GZK cut-off, and the chemical composition of cosmic rays. Indeed, evidence of UHECR, above the GZK cut-off, has been reported for the first time by the AGASA experiment [1]. On the contrary, the results of the HiRes [2] experiment and, more recently, the ones of the Pierre Auger Collaboration [3] are consistent with the existence of the cut-off. A key point which raises observing the cosmic ray energy spectra (Fig. 1) is the importance of the energy scale calibration between different experiments. It has been noted that with a shift by about $20 \%$ in the energy scale the disagreement between AGASA and HiRes results almost disappears. As can be seen from Fig. 1, a systematic difference from the previous measurements is present also for the Auger results. Many of the experimental procedure used to derive the energy spectra of the incoming cosmic rays depend strongly on the nuclear interaction models used in the Monte Carlo codes of the air showers, which amounts for the the main source of systematic uncertainties. To determine the nature of the cut-off and its astrophysical explanation, the determination of the absolute energy scale is indispensable, therefore reducing the uncertainty of the hadron interaction models would make an important contribution to resolve this problem of the absolute energy scale.
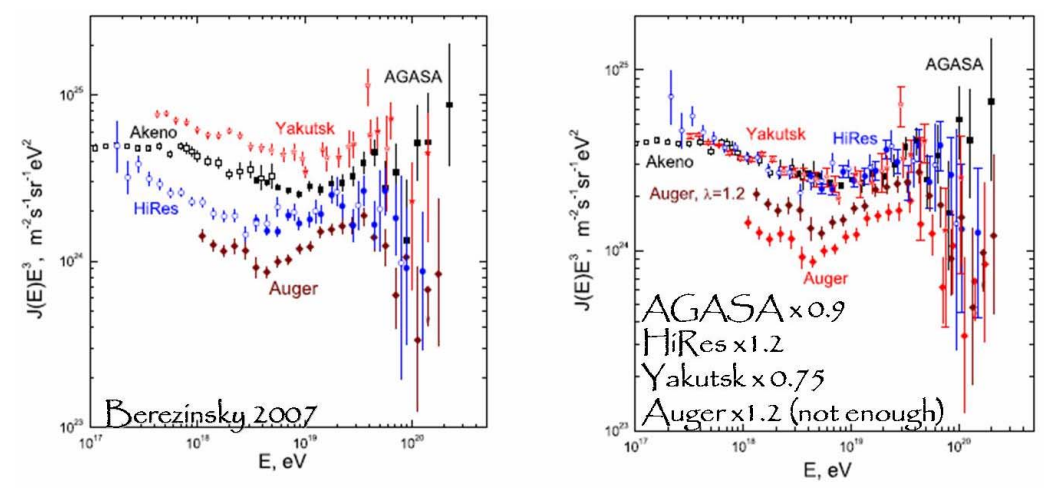

Figure 1: Energy spectra of cosmic rays at the highest energies. A comparison of the results from AGASA (black points), from HiRes (blue marks) and from AUGER (dark red) is shown. A clear discrepacy between AGASA, HiRes and Auger can be seen in the region above $10^{20} \mathrm{eV}$. In the right panel, the effect of a $20 \%$ adjustment on the energy scales is shown [4].

Similar considerations hold for the the interpretation of cosmic ray chemical composition. Cosmic rays are not purely protons but they contain also heavy nuclei. Nuclear cascade showers 
initiated by the disintegration of heavy nuclei develop more rapidly in comparison with the showers initiated by protons.

Recently, Auger results pose another puzzle for the highest energy extra-galactic cosmic rays. Auger results on the correlation between the arrival direction of the cosmic rays and the direction of AGNs seem to indicate that the highest energy cosmic rays are protons [5]. However, their results on air shower elongation seem to indicate that the composition of the highest energy cosmic-rays is likely dominated by heavy particles [6]. Because the deduction of primary cosmic ray composition from the elongation parameter has a strong model dependence, especially in the highest energy region, the reduction of the uncertainty in the interaction models is important for solving this puzzle. Once again accelerator experiments validating the interaction model chosen are essential. As a matter of fact air shower development is dominated by the forward products of the interaction between the primary particle and the atmosphere. The only available data on the production crosssection of neutral pions emitted in the very forward region have been obtained more than twenty years ago by the UA7 Collaboration [7] at CERN. They measured the photon distribution within an emission angle of as little as 1.8 milli-radians from the beam axis and up to an energy of 630 $\mathrm{GeV}$ in the center of mass system, corresponding to a laboratory frame energy of $2 \times 10^{4} \mathrm{eV}$, well below the knee region. The LHC accelerator, thanks to its unprecedented energy of $14 \mathrm{TeV}$ in the center of mass system, corresponding to $10^{17} \mathrm{eV}$ in the laboratory reference frame, offers a unique opportunity to meausure neutral particles emitted in the very forward region thus providing an essential tool to calibrate hadron interaction models used in cosmic ray measurements. The LHCf experiment at LHC has been proposed and build to achieve such important goal and is a fully dedicated LHC astroparticle experiment.

\section{The LHCf experiment}

The LHCf experiment is composed by two independent sampling calorimeters, called ARM1 and ARM2, placed on both side of the Interaction Point $1( \pm 140 \mathrm{~m})$ at the Large Hadron Collider (LHC), at CERN. The two calorimeters are housed in a beam absorbing structure (TAN) in which the two proton beams are steered in the two separate beam lines which circulate in the LHC machine. Thus the flux of charged particles is swept away by the magnets located between the Interaction Point and the TAN and only the neutral ones reach the calorimeter surface.

Each detector is arranged in a double tower geometry, each tower being made of plastic scintillators (16 layers) interleaved with tungsten layers as absorber. The two detectors have also position measurement capabilities thanks to the presence of four X-Y pairs of position sensitive layers: scintillation fibers (SciFi) and multianode PMTs in ARM1 and microstrip silicon sensors in ARM2. The lateral dimensions of the calorimeters range from $20 \mathrm{~mm} \times 20 \mathrm{~mm}$ to $40 \mathrm{~mm} \times 40 \mathrm{~mm}$ and the longitudinal size along the beam direction is $220 \mathrm{~mm}$. The geometry of the two detectors is slightly different both in dimension and tower structure: ARM1 has a two tower diamond structure, while in ARM2 the two towers are rotated by 90 degrees. The different geometrical arrangement allows, on one hand, to better control possible systematic effects and, on the other hand, the optimization for different running condition (beam crossing angle equal or different from zero and possible movements of the detector). A detailed description of the two detectors can be found in [8]. In 
Fig. 2 and Fig. 3 a schematic view of ARM1 detector and a photo of ARM2 detector are shown respectively.

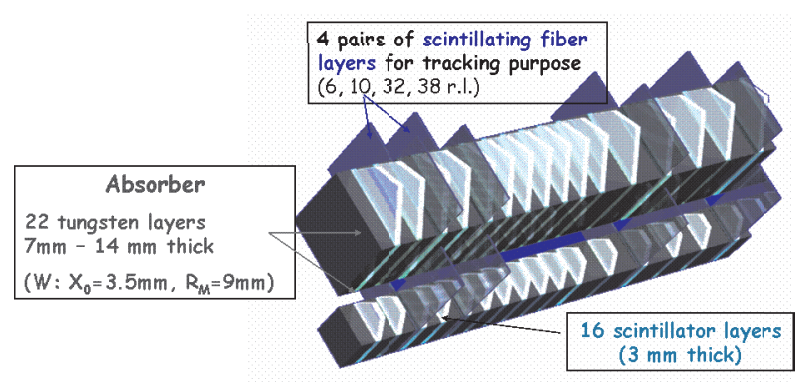

Figure 2: A schematic drawing of the ARM1 LHCf calorimeter. The two towers have transverse dimensions of $2 \times 2 \mathrm{~cm}^{2}$ and $4 \times 4 \mathrm{~cm}^{2}$. The scintillator tiles are read out with phototubes. Four layers of scintillation fibers are used for tracking purpose.

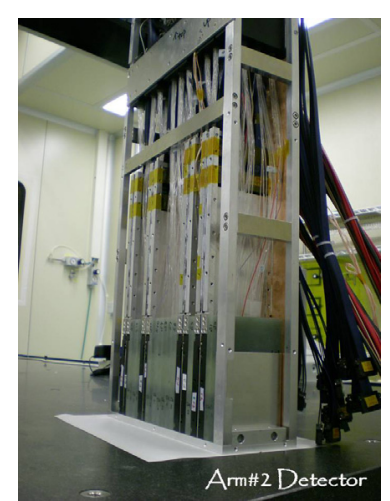

Figure 3: A photo of the ARM2 LHCf calorimeter. The two towers have transverse dimensions of $2.5 \times 2.5 \mathrm{~cm}^{2}$ and $3.2 \times 3.2$ $\mathrm{cm}^{2}$. For layers of silicon microstrip detectors with readout pitch of $160 \mu \mathrm{m}$ provide a high precision measurement of the shower profile.

The peculiar geometry consisting of two separate mini towers, is dictated by the need to reduce the shower leakage from one tower to the adjacent one due to the small size of the two towers. The leakage can however be corrected for by applying the position measurements of the silicon/scintillating fibre layers. In fact the position detectors are also used to select very clean sample of events where only one photon has showered in one of the towers. Also by reconstructing the two separate showers from the $2 \gamma$ from $\pi^{0}$ decays LHCf can obtain a high quality sample from which to measure the invariant mass and so calibrate the energy scale.

\section{Detector performances}

The performances of the two calorimeters have been extensively studied both with simulation studies and beam test data analysis.

At the end of August 2007 a beam test has been carried out on the final detectors using electron, muon and proton beams of different energies produced at the SPS CERN facility. This beam test has been devoted to calibrate the calorimeters and finally measure their performances in terms of energy resolution and spatial resolution of the sensitive layers. Fig. 4 shows the energy resolution measured with electrons of different energies for the ARM1 detector. The measured energy resolution is better than $4 \%$ at $200 \mathrm{GeV}$ in agreement with Monte Carlo expectations. Similar results have been obtained also for ARM2.

Fig. 5 shows the detector spatial resolution (i.e. the precision on the photon impact point measurement) for the ARM2 silicon position sensitive layer. It can be seen that the spatial resolution for a $200 \mathrm{GeV}$ electron is better than $40 \mu \mathrm{m}$. In Fig. 6 the energy dependence of the measured spatial resolution both for $\mathrm{X}$ and $\mathrm{Y}$ view is shown. Electrons of energies ranging from $50 \mathrm{GeV}$ up to 200 


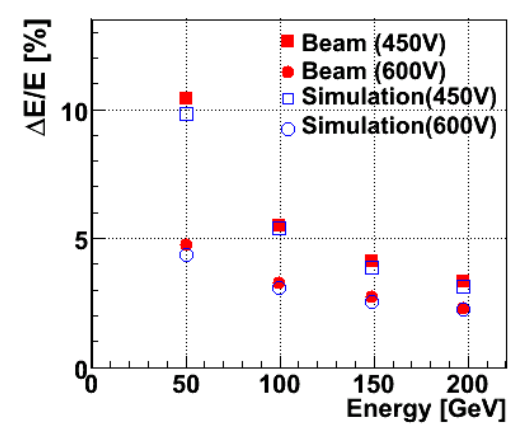

Figure 4: Energy resolution of ARM1 detector. Test beam results (red full marker) are in good agreement with simulation results (blue open marker). Data have been taken with two different PMT HV, corresponding to two different gain conditions.

$\mathrm{GeV}$ have been considered. The spatial resolution is below $100 \mu \mathrm{m}$ for energy grater than $100 \mathrm{GeV}$, which is the region of interest for LHCf, and improves for increasing energy. The measured resolution is in agrrement with simulation expectations and well satisfies the design requirement [8]. The resolution of the shower core for ARM1 detector is about $200 \mu \mathrm{m}$.

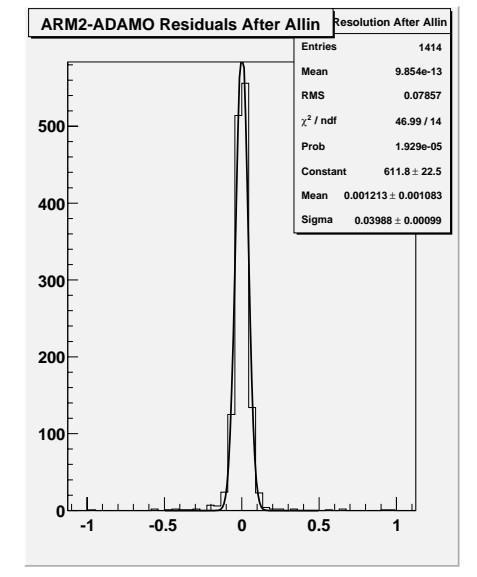

Figure 5: Position Resolution (in mm) of ARM2 silicon detector for $200 \mathrm{GeV}$ electrons.

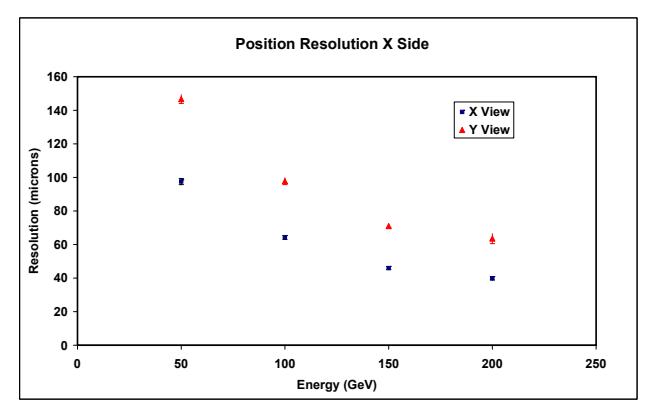

Figure 6: ARM2 silicon detector position resolution as function of energy. Resolution of $\mathrm{X}$ and $\mathrm{Y}$ view is shown.

During 2007 beam test the capability of the detector to reconstruct $\pi^{0}$ mass peak has also been measured. This is an important tool for the LHCf apparatus to calibrate the energy scale. A special set up has been arranged by placing a $6 \mathrm{~cm}$-thick carbon target in front of ARM1 on a $350 \mathrm{GeV}$ proton beam. Neutral pions produced in the interaction decay in two photons which in some cases are separately detected by the two towers which form the calorimeter. The results shown in Fig. 7 demonstrate that even with unfavourable experimental conditions (the energies of the photons produced with such set up is often well below the LHCf design lower limit of $100 \mathrm{GeV}$ and the contamination from primary protons are very high) the achievable $\pi^{0}$ mass resolution is 
less than $6 \%$ in good agreement with the 5\% Monte Carlo expectation [8].

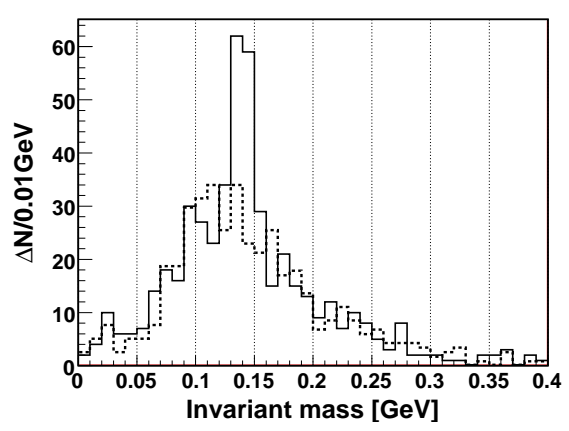

Figure 7: Reconstructed photon pair invariant mass distribution. The peak at the mass of the $\pi^{0}$ is clearly visible (the dotted histogram is the background calculated using uncorrelated photon pairs).

\section{Expected Physics Performances}

Waiting for the data taking, detailed simulation studies have been carried out to understand the capabilities of LHCf to disentangle different interaction models at LHC. Fig. 8 shows LHCf expectations for the $\gamma$ and neutron energy spectra. The models used are DPMJET3 [9], QGSJETII [10] and SYBILL [11]. Depending on the nuclear interaction model used the energy spectra change more or less significantly. In the case of $\gamma$ energy spectra, the discriminating power is already significant at $1 \mathrm{TeV}$ between SYBILL and the other codes, while discrimination between DPMJET3 and QGSJET-II can be achieved through a more sophisticated analysis, as described in Ref. [8].

The neutron sample gives more discrimination between different models. As it is shown in Fig. 8, even with a pessimistic $30 \%$ energy resolution, a very good disentangling of the different models is feasable.
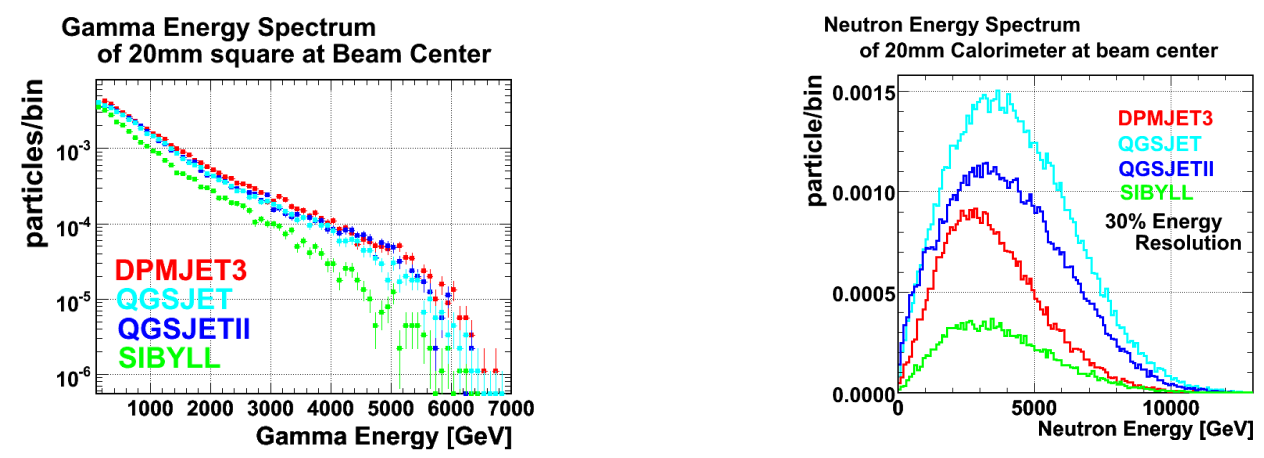

Figure 8: Expected energy spectrum for $\gamma_{\mathrm{s}}$ and neutrons according to different interaction models. For neutrons a $30 \%$ energy resolution has been taken into account.

Recently new models have been proposed $[12,13]$ especially to describe the muon production in cosmic rays. The measurements of the LHCf experiment will be extremely helpful to calibrate these models hence to provide also important constraints for muon production in air showers. 


\section{Conclusions}

The LHCf detectors have been successfully installed in the LHC tunnel since January 2008. The detectors are ready for the data taking, the DAQ and Slow Control system have been extensively tested and during the first LHC beam circulation, first data in the Frount Counter detectors have been collected. LHCf will start its measurements during the beam-commissioning phase foreseen to restart in summer 2009 and will take data to complete the first part of its physics program with the 7+7 TeV runs. The LHCf experiment will hence be capable to calibrate air shower Monte Carlo codes up to the energy of $10^{17} \mathrm{eV}$, thus providing invaluable input to questions posed since the first detection of UHECR.

\section{References}

[1] M. Takeda et al., Extension of the cosmic ray energy spectrum beyond the predicted Greisen-Zatsepin-Kuzfmin cutoff, Phys. Rev. Lett. 81 (1998) 1163 [astro-ph/9807193].

[2] R. U. Abbasi et al., Measurement of the Flux of Ultrahigh Energy Cosmic Rays from Monocular Observations by the High Resolution Fly's Eye Experiment, Phys. Rev. Lett. 92 (2004) 1511.

[3] T. Yamamoto et al., The UHECR spectrum measured at the Pierre Auger Observatory and its astrophysical implications, Proc. of 30th Intl. Cosmic Ray Conference, Merida, Mexico (2007) [arXiv:0707.2638 [astro-ph] ].

[4] V. Berezinsky, Transition from galactic to extragalactic cosmic rays, Proc. of 30th Intl. Cosmic Ray Conference, Merida (2007) [arXiv: 0710.2750v2 [astro-ph] ]

[5] The Pierre Auger Collaboration, Correlation of the Highest Energy Cosmic Rays with nearby Extragalactic Objects, Science 318 (2007) 938.

[6] M. Unger et al., Study of the Cosmic Ray Composition above 0.4 EeV using the Longitudinal Profiles of Showers observed at the Pierre Auger Observatory, Proc. of 30th Intl. Cosmic Ray Conference, Merida (2007) [arXiv:0706.1495v1 [astro-ph] ]

[7] E. Paré et al., Inclusive production of $\pi^{0}$ s in the fragmentation region at the SppS collider, Phys. Lett. B 242 (1990) 531.

[8] O. Adriani et al., Technical design report of the LHCf experiment, CERN-LHCC-2006-004.

[9] S. Roesler et al., The Event generator DPMJET-III at cosmic ray energies, Proc. of 27th International Cosmic Ray Conference (ICRC2001), Hamburg (2001).

[10] S. Ostapchenko, Status of QGSJET, AIP Conf. Proc. 928 (2007) 118 [arXiv: 0706.3784 [astro-ph] ].

[11] R. S. Fletcher et al., SIBYLL: An Event generator for simulation of high-energy cosmic ray cascades, Phys. Rev. D 50 (1994) 5710.

[12] K. Werner and T. Pierog, Extended Air Shower Simulations Based on EPOS, AIP Conf. Proc. 928 (2007) 111 [arXiv:0707.3330 [astro-ph] ].

[13] H. J. Drescher, Remnant Break-up and Muon Production in Cosmic Ray Air Showers, Physical Review D 77, (2008) 056003 [arXiv: 0712 .1517v1 [hep-ph] ]. 